\title{
Preditores da mortalidade materna por COVID-19: revisão integrativa
}

\author{
Predictors of maternal mortality by COVID-19: an integrative review \\ Predictores de mortalidad materna por COVID-19: una revisión integradora
}

Recebido: 29/07/2021 | Revisado: 07/08/2021 | Aceito: 11/08/2021 | Publicado: 15/08/2021

\author{
Isabela de Lima da Silva \\ ORCID: https://orcid.org/0000-0002-7207-8072 \\ Universidade Federal do Rio Grande do Norte, Brasil \\ E-mail: Isabelalimaej@gmail.com \\ Ravana Amália Ribeiro Barreto \\ ORCID: https://orcid.org/0000-0003-2677-8198 \\ Universidade Federal do Rio Grande do Norte, Brasil \\ E-mail: ravanaamalia@hotmail.com \\ Brenda Kelly Pontes Soares \\ ORCID: https://orcid.org/0000-0001-7873-1653 \\ Universidade Federal do Rio Grande do Norte, Brasil \\ E-mail: brendaa.pontes@gmail.com \\ Allyne Dantas Matias \\ ORCID: https://orcid.org/0000-0003-2888-9465 \\ Universidade Federal do Rio Grande do Norte, Brasil \\ E-mail: allynedantasmatias@hotmail.com \\ Francisca Marta de Lima Costa Souza \\ ORCID: https://orcid.org/0000-0002-2442-9499 \\ Universidade Federal do Rio Grande do Norte, Brasil \\ E-mail: enfermarta2001@yahoo.com.br
}

\begin{abstract}
Resumo
Objetivo: revisar a produção científica os fatores relacionados à mortalidade materna causada pela infecção da COVID-19. Métodos: Trata-se de uma revisão integrativa. O levantamento das publicações ocorreu maio de 2021 utilizando os descritores "Mortalidade Materna", "COVID-19" e "Gravidez", na Biblioteca Virtual em Saúde (BVS). Após análise dos estudos, quatro artigos foram incluídos como amostra final. Resultados: As complicações e evolução para óbito em gestantes contaminadas pela COVID-19, estão ligadas a associação das alterações fisiológicas do processo gravídico, as comorbidades como as doenças cardiovasculares, obesidade e diabetes; assistência inadequada no pré-natal, pós-parto e no serviço avançado (UTI e apoio ventilatório), além das questões raciais. Conclusão: A identificação de alguns fatores que agravam o estado de saúde das gestantes contaminadas pela COVID-19, podendo gerar complicações fatais, uma vez que, as próprias alterações fisiológicas e anatômicas do período gestacional aliadas as comorbidades decorrentes das doenças crônicas, predispõe ao maior risco de hospitalizações. Faz-se necessário ações de educação permanente para que a oferta do serviço seja prestada de forma especializada, eficaz e com equidade, afim de atenuar os riscos de mortes em gestantes.
\end{abstract}

Palavras-chave: Gravidez; COVID-19; Mortalidade.

\begin{abstract}
Objective: to review scientific production on factors related to maternal mortality caused by COVID-19 infection. Methods: This is an integrative review. The survey of publications took place in May 2021 using the descriptors "Maternal Mortality", "COVID-19" and "Pregnancy", in the Virtual Health Library (VHL). After analyzing the studies, four articles were included as the final sample. Results: Complications and evolution to death in pregnant women contaminated by COVID-19 are linked to the association of physiological changes in the pregnancy process, comorbidities such as cardiovascular disease, obesity and diabetes; inadequate assistance in prenatal, postpartum and advanced service (ICU and ventilatory support), in addition to racial issues. Conclusion: The identification of some factors that aggravate the health status of pregnant women contaminated by COVID-19, which can lead to fatal complications, since the physiological and anatomical changes of the gestational period, combined with comorbidities resulting from chronic diseases, predispose to greater risk of hospitalizations. Continuing education actions are needed so that the service is provided in a specialized, effective and equitable manner, in order to mitigate the risk of death in pregnant women.
\end{abstract}

Keywords: Pregnancy; COVID-19; Mortality.

\section{Resumen}

Objetivo: revisar la producción científica sobre factores relacionados con la mortalidad materna causada por la infección por COVID-19. Métodos: esta es una revisión integradora. La encuesta de publicaciones se realizó en mayo 
de 2021 utilizando los descriptores "Mortalidad materna", "COVID-19" y "Embarazo", en la Biblioteca Virtual en Salud (BVS). Tras analizar los estudios, se incluyeron cuatro artículos como muestra final. Resultados: Las complicaciones y evolución a muerte en gestantes contaminadas por COVID-19 están vinculadas a la asociación de cambios fisiológicos en el proceso de gestación, comorbilidades como enfermedad cardiovascular, obesidad y diabetes; Asistencia inadecuada en el servicio prenatal, posparto y avanzado (UCI y soporte ventilatorio), además de cuestiones raciales. Conclusión: La identificación de algunos factores que agravan el estado de salud de las gestantes contaminadas por COVID-19, que pueden derivar en complicaciones fatales, ya que los cambios fisiológicos y anatómicos del período gestacional, combinados con las comorbilidades derivadas de enfermedades crónicas, predisponen a una mayor riesgo de hospitalizaciones. Se requieren acciones de educación permanente para que el servicio se brinde de manera especializada, efectiva y equitativa, con el fin de mitigar el riesgo de muerte en mujeres embarazadas.

Palabras clave: Embarazo; COVID-19; Mortalidad.

\section{Introdução}

O vírus da COVID-19 possui como agente etiológico o SARS-CoV-2, sendo caracterizado por ser uma síndrome respiratória aguda (SRAG) que cresce em progressão exponencial e rápida. O primeiro caso oficial da doença no mundo surgiu em dezembro de 2019 na cidade de Wuhan, localizada na China. No dia 11 de março de 2020, a Organização Mundial de Saúde (OMS) decretou pandemia mundial pelo novo coronavírus, levantando a necessidade da implementação de medidas específicas para identificação, prevenção e controle do vírus (Campos et al., 2020; Nascimento et al., 2021).

A principal forma de transmissão da COVID-19 é por meio do contato próximo com pessoas infectadas ou objetos e superfícies contaminados, acarretando danos imensuráveis a saúde. Além disso, alguns indivíduos são considerados mais susceptíveis a infecção, classificados como grupos de riscos, entre esses estão: idosos, portadores de comorbidades como diabetes e hipertensão e gestantes (Guan et al., 2020; Nascimento et al., 2021).

Os primeiros estudos traziam hipóteses que a infecção por COVID-19 era semelhante a H1N1, sendo, portanto, mais grave em gestantes. Todavia, outras pesquisas iniciais evidenciavam que o coronavirus ocorria com a mesma frequência e gravidade em gestantes e não gestantes, e assim a preocupação estava voltada mais para as gravidas de alto risco acometidas por doenças como diabetes, obesidade e hipertensão (Chen et al., 2020; Sutton, Fuchs, D'Alton, \& Goffman.., 2020).

Em abril de 2020, o Ministério da Saúde (MS) lança a Nota Técnica No 12/2020 com o objetivo de evidenciar os riscos do COVID-19 às gestantes e puérperas (Brasil, 2020). Um estudo realizado por Kadiwar et al. (2021) mostra que em setembro de 2020, após uma revisão sistemática e meta-análise de dados globais, foi reconhecido que a gravidez é um fator de risco para uma apresentação mais grave do vírus. Estudos apontam a indispensabilidade do conhecimento acerca das repercussões causada pela COVID-19 durante o período gestacional e puerperal, já que os mesmos evidenciam que o segundo e terceiro trimestre, e/ou no puerpério, são períodos de maior probabilidade de complicações que podem acarretar óbito materno. (Barbosa et al., 2021).

O Brasil é uma das lideranças no número de casos de mortes maternas por COVID-19, relatando que de cada 10 óbitos no mundo, 8 eram no país. Além disso, possui uma taxa de letalidade de 7,2\%, onde até 26 de maio de 2021 foram registrados 911 óbitos maternos pela doença. (Pereira et al., 2020; Brasil, 2021).

O MS em conjunto com a Organização Pan-Americana de Saúde (OPAS) lança o Manual de Recomendações para a Assistência à gestante e Puérpera com o objetivo de nortear e melhorar o acesso e a assistência a essas mulheres durante o período de pandemia em todo o seu ciclo gravídico-puerperal, abordando acerca das vias de transmissão, diagnóstico e tratamento da doença em suas diversas fases de infecção, objetivando reduzir os possíveis agravos e mortalidade materna e fetal. (Brasil, 2020).

Neste contexto, sabendo que o vírus da COVID-19 pode causar agravos a saúde materno-fetal, em alguns casos resultando em morte, torna-se importante aprofunda-se o conhecimento acerca do assunto buscando alternativas para reduzir 
esse problema. Diante desta problemática, o objetivo do estudo foi identificar na literatura científica os fatores relacionados à mortalidade materna causada pela infecção da COVID-19.

\section{Metodologia}

Trata-se de uma revisão integrativa, elaborada em seis etapas: formação da pergunta norteadora; amostragem; coleta de dados; análise dos estudos incluídos; discussão dos resultados e a apresentação da revisão.

A questão norteadora se desenvolveu a partir da estratégia PICO - População: Gestantes; Interesse: Artigos científicos; Contexto: Infecção COVID-19; Outcome/Desfecho: Morte materna. Assim, elaborou-se a questão da pesquisa: “Quais fatores relacionados à mortalidade materna causada pela infecção da COVID-19?” (Mendes, Silveira, \& Galvão, 2008)

A pesquisa dos dados ocorreu no mês de maio de 2020, a partir da Biblioteca Virtual em Saúde (BVS), utilizando os descritores identificados pelos Descritores em Ciências da Saúde (DECs). Na estratégia de busca o operador booleano utilizado foi: "Mortalidade Materna” AND “COVID-19” AND “Gravidez”.

Os critérios de inclusão adotados foram: artigos de pesquisa originais, que atendessem a temática e open access. Adotou-se como critérios de exclusão os estudos que não tinham metodologia de pesquisa (relatos de caso, reflexões, recomendações), revisões e literatura cinzenta (teses, dissertações, documento, relatos de caso, carta e livros).

Para a seleção dos estudos utilizados na pesquisa seguiu-se a recomendação Preferred Reporting Items for Systematic Reviews and Meta-Analyses (PRISMA), conforme apresentado no Fluxograma 1.

Fluxograma 1 - Seleção dos estudos com base na recomendação PRISMA. Santa Cruz, Rio Grande do Norte, Brasil, 2021.

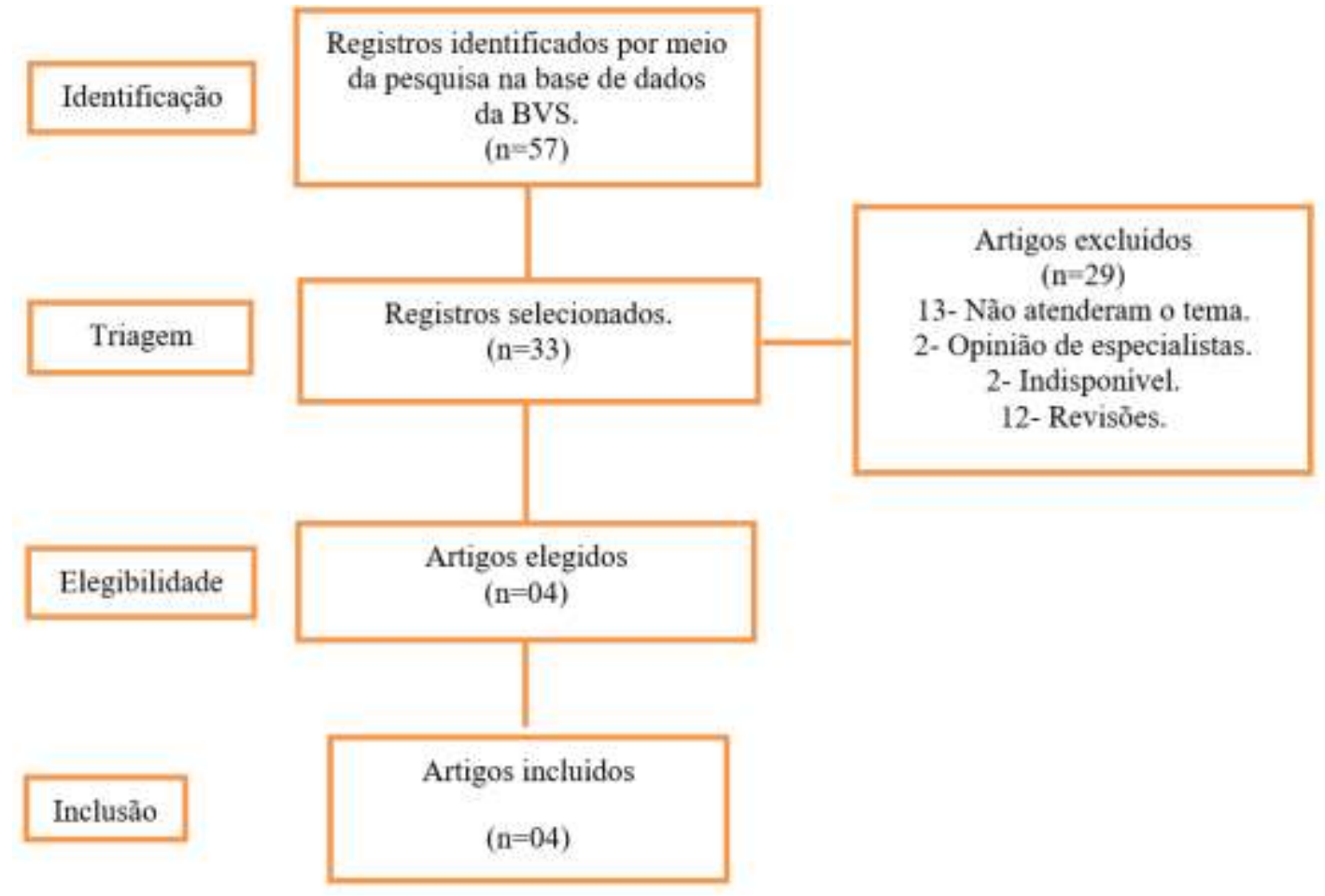

Fonte: Autores (2021).

\section{Resultados}

$\mathrm{Na}$ análise dos quatro artigos selecionados para este estudo, em relação à amostra, todos foram realizados a partir de dados secundários concentrados no período de 2020. Quanto ao continente, um estudo na América do Norte, América do Sul, América Central e Europa, respectivamente. Já o idioma, todos na língua inglesa. 
No tocante ao nível de evidência, a classificação foi dada de acordo com a abordagem metodológica, atribuindo-se: Nível I - Revisão Sistemática ou Metanálise de Ensaios Clínicos Controlados e Randomizados, nível II - Ensaio Clínico Controlado e Randomizado com Intervalo de Confiança Estreito, nível III - resultados terapêuticos e ensaios clínicos não randomizados, nível IV - Estudos de caso-controle e estudos de coorte, nível V - revisão sistemática de estudos qualitativos, descritivos e filosóficos, nível VI - estudo individual de caráter descritivo e qualitativo, nível VII - opiniões de especialistas e autoridades (Stetler et al., 1998). Dessa forma, na presente pesquisa identificou-se que os quatro estudos se classificam no nível IV. A tabela 1 mostra a síntese dos artigos incluídos.

Tabela 1 - Artigos segundo autoria, revista, objetivo, método de pesquisa e principais resultados. Santa Cruz, Rio Grande do Norte, Brasil, 2021.

\begin{tabular}{|c|c|c|c|c|}
\hline AUTOR & REVISTA & OBJETIVO & MÉTODO & PRINCIPAIS RESULTADOS \\
\hline 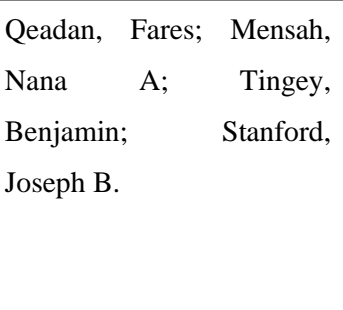 & $\begin{array}{l}\text { BMC } \\
\text { Pregnancy } \\
\text { Childbirth }\end{array}$ & $\begin{array}{l}\text { Avaliar o risco ajustado das } \\
\text { seguintes complicações da } \\
\text { COVID-19 em relação à } \\
\text { gravidez: hospitalização, } \\
\text { tempo máximo de internação, } \\
\text { ventilação e óbito. }\end{array}$ & $\begin{array}{l}\text { Estudo } \\
\text { Retrospectivo }\end{array}$ & $\begin{array}{l}\text { Verificou-se que entre as mulheres com doença } \\
\text { COVID-19, a gravidez confere risco adicional } \\
\text { substancial de morbidade, mas nenhuma diferença } \\
\text { na mortalidade. }\end{array}$ \\
\hline $\begin{array}{l}\text { Di Guardo, Federica; Di } \\
\text { Grazia, Flavia Maria; Di } \\
\text { Gregorio, Luisa Maria; } \\
\text { Zambrotta, Elisa; Carrara, } \\
\text { Grazia; Gulino, Ferdinando } \\
\text { Antonio; Tuscano, Attilio; } \\
\text { Palumbo, Marco }\end{array}$ & $\begin{array}{l}\text { Arch } \\
\text { Gynecol } \\
\text { Obstet }\end{array}$ & $\begin{array}{l}\text { Analisar a gravidade dos } \\
\text { desfechos materno-neonatais } \\
\text { em gestantes afetadas pela } \\
\text { SARS-CoV-2. }\end{array}$ & $\begin{array}{l}\text { Estudo } \\
\text { Observacional }\end{array}$ & $\begin{array}{l}\text { Analisou-se que em relação ao tipo de parto, o } \\
\text { percentual de pacientes com parto normal foi } \\
\text { maior do que com parto cesáreo. Enfatizou-se que } \\
\text { a maioria dos casos de morte materna ocorreu em } \\
\text { pacientes com sintomas graves. }\end{array}$ \\
\hline $\begin{array}{l}\text { Ríos-Silva, } \\
\text { Murillo-Zamora, } \quad \text { Efrén; } \\
\text { Mendoza-Cano, } \\
\text { Trujillo, Xóchitiver; } \\
\text { Miguel }\end{array}$ & $\begin{array}{l}\text { J Glob } \\
\text { Health }\end{array}$ & $\begin{array}{l}\text { Identificar fatores de risco } \\
\text { para complicações e óbito em } \\
\text { mulheres em idade fértil e } \\
\text { gestantes com suspeita de } \\
\text { COVID-19 }\end{array}$ & $\begin{array}{l}\text { Estudo } \\
\text { Retrospectivo }\end{array}$ & $\begin{array}{l}\text { Pacientes grávidas não apresentam maior risco de } \\
\text { complicações ou morte por COVID-19 do que } \\
\text { pacientes não grávidas. A presença de Diabetes } \\
\text { Mellitus e doenças crônicas aumentam o risco de } \\
\text { morte em mulheres em idade fértil, mas não } \\
\text { especificamente em pacientes grávidas. }\end{array}$ \\
\hline $\begin{array}{l}\text { Takemoto, Mls; Menezes, } \\
\text { M O; Andreucci, C B; } \\
\text { Knobel, R; Sousa, L; Katz, } \\
\text { L; Fonseca, E } \mathrm{B} \text {; } \\
\text { Nakamura-Pereira, } \\
\text { Magalhães, C G; Diniz, } \\
\text { Csg; Melo, Aso; Amorim, } \\
\text { Mmr. }\end{array}$ & BJOG & $\begin{array}{l}\text { Descrever as características } \\
\text { clínicas de mulheres grávidas } \\
\text { e puérperas com COVID-19 } \\
\text { grave no Brasil e examinar os } \\
\text { fatores de risco para } \\
\text { mortalidade. }\end{array}$ & $\begin{array}{l}\text { Estudo } \\
\text { Transversal }\end{array}$ & $\begin{array}{l}\text { Identificou-se que pelo menos uma comorbidade } \\
\text { estava presente nos casos fatais em comparação } \\
\text { aos casos de sobrevivência. Entre as mulheres que } \\
\text { morreram, a maioria necessitou de hospitalização } \\
\text { em UTI necessitando de suporte ventilatório } \\
\text { invasivo. Os principais fatores de risco para morte } \\
\text { materna por COVID-19 foram o pós-parto no } \\
\text { início da Síndrome do Desconforto Respiratório } \\
\text { Agudo, obesidade, Diabetes Mellitus e doenças } \\
\text { cardiovasculares, assim como mulheres negras } \\
\text { tiveram maior prevalência de óbito. }\end{array}$ \\
\hline
\end{tabular}


A partir da análise dos artigos, observaram-se alguns fatores preditores associados à mortalidade materna ocasionada pela infecção do vírus da COVID-19.

\section{Discussão}

Alguns estudos publicados demonstraram que a presença de uma comorbidade em pacientes diagnosticados com COVID-19, estão associados a maiores complicações da doença e com desfechos insatisfatórios (Qeadan et al., 2021). Como forma de diminuir complicações e mortes, foram identificados os grupos de riscos, dos quais as gestantes fazem parte. As mulheres grávidas estão associadas ao grupo de risco pelas alterações anatômicas e fisiológicas da gravidez, como cardiovasculares, respiratórias e imunológicas, que as tornam mais susceptíveis a pneumonias virais (Souza, \& Amorim, 2021).

De acordo com os achados dos dados da amostra, as mulheres grávidas com diagnóstico de COVID-19 tinham mais chances de serem hospitalizadas e necessitarem de ventilação moderada, com maior permanência durante a hospitalização do que as mulheres não grávidas hospitalizadas por COVID-19 (Qeadan, Mensah, Tingey, \& Stanford, 2021). O relatório do Centro de Controle de Doenças dos EUA e estudos realizados na Suécia, descrevem uma maior suscetibilidade às complicações da doença em mulheres grávidas, como também o aumento na hospitalização, admissão na unidade de terapia intensiva (UTI) e ventilação mecânica, mas não foi associado a casos de mortalidade materna (Guardo et al., 2021; Takemoto et al., 2020a; Souza \& Amorim, 2021; Takemoto et al., 2020b).

Nos dados analisados na planilha do Sistema de Informações de Vigilância Epidemiológica da Gripe (SIVEP Gripe), do Ministério da Saúde, foram identificados 978 diagnósticos da Síndrome Respiratória Aguda Grave (SRAG) por COVID-19 em gestantes e puérperas, dentre estas, 124 foram óbitos, 12,7\% de letalidade (Souza \& Amorim, 2021). Esses óbitos foram associados a comorbidades, como doenças cardiovasculares, obesidade e diabetes. Além disso, foi identificado graves falhas durante a assistências a essas mulheres, como na assistência ventilatória, acesso a leito na UTI e a não realização da intubação e ventilação mecânica (Takemoto et al., 2020a; Qeadan et al., 2021; Souza \& Amorim, 2021).

O puerpério também foi citado na literatura com uma maior frequência de mortes e sugerem que a mortalidade em gestantes no Brasil se justifica pelas causas de doenças crônicas, recursos insuficientes, pouca qualidade no pré-natal, leitos indisponíveis, dificuldade no acesso aos serviços de saúde, racismo e violência obstétrica (Takemoto et al., 2020a; Qeadan et al., 2021; Souza \& Amorim, 2021; Takemoto et al., 2020b).

As diferenças étnicas, raciais e minoritárias, tem um aumento crescente na mortalidade materna em decorrência da doença do coronavírus. Mulheres negras tem uma maior hospitalização em condições mais graves, como dispneia e menor saturação de oxigênio, além de menor admissão na UTI e ventilação mecânica, com maiores riscos de morte comparadas as mulheres brancas. Isso ocorre por maiores prevalências de comorbidades, condições de trabalhos ruins, habitação, condições de vida e racismo estrutural, pois são motivos observados nas populações minoritárias (Qeadan et al., 2021; Souza \& Amorim, 2021).

Existe uma lacuna nesses cuidados, em decorrência do pré-natal que foi afetado durante o período da pandemia, que pode ser explicado por possíveis falhas do município ao prestar assistência prioritária ao COVID-19, ou ao medo e incertezas das gestantes ao sair de caso, acarretando o aumento da ansiedade e depressão. Diante dos achados, é imprescindível o cuidado integral e de qualidade às gestantes e puérperas, sem que ocorra a discriminação racial, atentando para suas necessidade e demandas. Dessa forma, diminuindo os riscos de morbimortalidades. Ofertando assim, a promoção, proteção, tratamento adequado e a recuperação da saúde a essas gestantes (Souza \& Amorim, 2021). 


\section{Conclusão}

O estudo colaborou com a identificação de alguns fatores que agravam o estado de saúde das gestantes contaminadas pela COVID-19, podendo gerar complicações fatais, uma vez que, as próprias alterações fisiológicas e anatômicas do período gestacional aliadas as comorbidades decorrentes das doenças crônicas, predispõe ao maior risco de hospitalizações. Além disso, identificou-se a assistência inadequada tanto no acompanhamento do pré-natal ao pós-parto da mulher contaminada, quanto no serviço especializado para suporte ventilatório e UTI. Fazem-se necessárias assim, ações de educação permanente para os profissionais que atuam diretamente com essas pacientes, para assim, minimizar os agravos oriundos de tais lacunas assistenciais.

Ademais, foi apontado que mulheres negras estão mais suscetíveis ao agravo da COVID-19, uma vez que há associação da prevalência de comorbidades ao racismo estrutural, que é dado como determinante social de saúde.

O estudo é relevante para a área da enfermagem por se tratar do campo de prática dessa profissão, como para outros profissionais que trabalham na linha de frente ao combate do coronavirus, favorecendo o conhecimento sobre essa temática e divulgação científica.

Observou-se como limitações para a presente pesquisa, a escassez de estudos, visto que a temática é nova em virtude do tempo de pandemia e as variações epidemiológicas. Ressalta-se, assim a importância de um enfoque maior á temática, tendo em vista sua complexidade e impacto na vida da população.

\section{Referências}

Barbosa, A. C. S., Costa, C. M. da., Santos, D. T. da S., Fonseca, I. C. V., Freitas, L. C. D., Fonseca, L. B., Rezende, C. A., Bernardo, G. J. R., \& Oliveira, M M. (2021). Repercussions in newborns infected by the new coronavirus during pregnancy: Integrative review. Research, Society and Development, 10(8), e31510817364. https://doi.org/10.33448/rsd-v10i8.17364

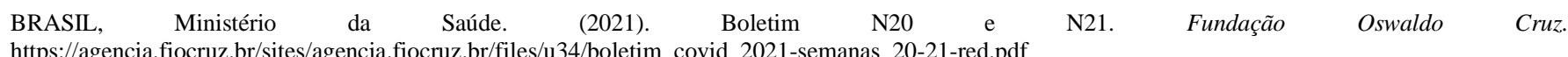

BRASIL. Ministério da Saúde. Nota Informativa nº 13/2020 - SE/GAB/SE/MS. Manual de Recomendações para a Assistência à Gestante e Puérpera frente à Pandemia de Covid-19. https://www.gov.br/saude/pt-br/coronavirus/publicacoes-tecnicas/guias-e-planos/manual-instrutivo-para-a-assistencia-a-gestante-epuerpera-frente-a-pandemia-da-covid-19

BRASIL. Ministério da Saúde. Nota Técnica $\mathrm{n}^{\mathrm{a}}$ 12/2020-COSMU/CGCIVI/DAPES/SAPS/MS. Infecção COVID-19 e os riscos às mulheres no ciclo gravídico-puerperal. https://portaldeboaspraticas.iff.fiocruz.br/wp-content/uploads/2020/04/SEI_MS-0014496630-Nota-T\%C3\%A9cnica-4_18.04.2020.pdf

Campos, M. R., Schramm, J. M. A., Emmerick, I. C. M., Rodrigues, J. M., Avelar, F. G., \& Pimentel, T. G. (2020). Carga de doença da COVID-19 e de suas complicações agudas e crônicas: reflexões sobre a mensuração (daly) e perspectivas no sistema único de saúde. Cadernos de Saúde Pública,. 36(11), 1-14. https://www.scielo.br/j/csp/a/bHbdPzJBQxfwkwKWYnhccNH/abstract/?lang=pt. DOI: https://doi.org/10.1590/0102-311X00148920

Chen, H., Guo, J., Wang, C., Luo, F., Yu, X., Zhang, W., Li, J., et. al. (2020). Clinical characteristics and intrauterine vertical transmission potential of COVID-19 infection in nine pregnant women: a retrospective review of medical. The Lancet, 395(10226), 1-7. https://www.thelancet.com/journals/lancet/article/PIIS0140-6736(20)30360-3/fulltext\#. DOI: https://doi.org/10.1016/S0140-6736(20)30360-3

Guan, W., Ni, Z., Yu Hu, W., Liang, C. O., He, J., Liu, L., et. al. (2021). Clinical Characteristics of Coronavirus Disease 2019 in China. New England Journal Of Medicine, 303(18), https://www.nejm.org/doi/full/10.1056/nejmoa2002032. DOI: 10.1056 / NEJMoa2002032

Guardo, F. D., Grazia, F. M. D., Gregorio, L. M. D., Zambrotta, E., Carrara, G., Gulino, F. A., Tuscano, A. et. al. Poor maternal-neonatal outcomes in pregnant patients with confirmed SARS-Cov-2 infection: analysis of 145 cases. Archives Of Gynecology And Obstetrics, 303(6), 1483-1488. https://link.springer.com/article/10.1007/s00404-020-05909-4\#citeas. DOI: https://doi.org/10.1007/s00404-020-05909-4

Kadiwar, S., Smith, J. J., Ledot, S., Johnson, M., Bianchi, P., Singh, N., Montanaro, C., et. al. (2021). Were pregnant women more affected by COVID-19 in the second wave of the pandemic? The Lancet, 397(10284), 1-2. https://www.thelancet.com/journals/lancet/article/PIIS0140-6736(21)00716-9/fulltext\#. DOI: https://doi.org/10.1016/S0140-6736(21)00716-9

Mendes, K. D. S., Pereira Silveira, R. C. C., \& Galvão, C. M. (2008). Revisão integrativa: método de pesquisa para a incorporação de evidências na saúde e na enfermagem. Texto contexto -enferm, 17(4), 1-7. http://www.scielo.br/scielo.php?script=sci_arttext\&pid=S0104-07072008000400018\&lng=en. DOI: https://doi.org/10.1590/S0104-07072008000400018

Nascimento, JM, Silva, APM da, Stuart, MR, Pereira Junior, AC, Pimentel, B., Souza, ML da SP de, \& Costa, R. (2021). O protagonismo da enfermagem em um centro cirúrgico / obstétrico COVID-19 nas adaptações assistenciais: Relato de experiência. Research, Society and Development , 10 (8), e19210817307. https://doi.org/10.33448/rsd-v10i8.17307 
Research, Society and Development, v. 10, n. 10, e435101018888, 2021

(CC BY 4.0) | ISSN 2525-3409 | DOI: http://dx.doi.org/10.33448/rsd-v10i10.18888

Pereira, M. N., Amorim, M. M. R., Pacagnella, R. C., Takemoto, M. L. S., Penso, F. C. C., Filho, J. R., \& Leal, M. C. (2020). COVID-19 and Maternal Death in Brazil: An Invisible Tragedy. Revista Brasileira Ginecolia Obstetrica, 42(8), 445-447. Retrieved 10 maio, 2021, from https://pubmed.ncbi.nlm.nih.gov/32898910/. DOI: 10.1055 / s-0040-1715138

Qeadan, F., Mensah, N. A., Tingey, B., \& Stanford, J. B. (2021). The risk of clinical complications and death among pregnant women with COVID-19 in the Cerner COVID-19 cohort: a retrospective analysis. Bmc Pregnancy And Childbirth, 21(1), 1-14. https://bmcpregnancychildbirth.biomedcentral.com/articles/10.1186/s12884-021-03772-y\#citeas. DOI: https://doi.org/10.1186/s12884-021-03772-y

Souza, A. S. R., \& Amorim, M. M. R. (2021). Maternal mortality by COVID-19 in Brazil. Revista Brasileira de Saúde Materno Infantil, 21(1), S253- S256. https://www.scielo.br/j/rbsmi/a/R7MkrnCgdmyMpBcL7x77QZd/?lang=en\#. DOI: https://doi.org/10.1590/1806-9304202100S100014

Stetler, C. B., Morsi, D., Rucki, S., Broughton, S., Corrigan, B., Fitzgerald, J., Giuliano, K., et. al. (1998). . Utilization-focused integrative reviews in a nursing service. Appl Nurs Re, 11(4), 195-206. https://pubmed.ncbi.nlm.nih.gov/9852663/. DOI: 10.1016 / s0897-1897 (98) 80329-7

Sutton, D., Fuchs, K., D'Alton, M., \& Goffman, D. (2020). Universal Screening for SARS-CoV-2 in Women Admitted for Delivery. New England Journal Of Medicine, 38(22), 2163- 2164. https://www.nejm.org/doi/full/10.1056/NEJMc2009316. DOI: 10.1056 / NEJMc2009316

Takemoto, M. L. S., Menezes, M.O., Andreucci, C. B., Knobel, R., Sousa, L., Katz, L., Fonseca, E. B., et. al. (2020a). Maternal deaths in Brazil from severe COVID-19 respiratory disease: time for a global commitment to ending health disparities. Bjog: An International Journal of Obstetrics \& Gynaecology, 127(19), 1-9. https://obgyn.onlinelibrary.wiley.com/doi/10.1111/1471-0528.16470. DOI: https://doi.org/10.1111/1471-0528.16470

Takemoto, M. L. S., Menezes, M. O., Andreucci, C. B., Pereira, M. N., Amori, M. M. R., Katz, Leila., \& Knobel, R. (2020b). The tragedy of COVID-19 in Brazil: 124 maternal deaths and counting. International Journal Of Gynecology \& Obstetrics, 151(1), $154-156$. https://obgyn.onlinelibrary.wiley.com/doi/10.1002/ijgo.13300. DOI: https://doi.org/10.1002/ijgo.13300 